\title{
Detector R\&D for future neutrino oscillations facilities
}

\author{
F.J.P. Soler*it \\ Department of Physics and Astronomy, University of Glasgow, Glasgow, G12 8QQ, UK. \\ E-mail: p.solerephysics.gla.ac.uk
}

This article describes the detector requirements for future neutrino oscillation facilities, the development of the software and the analysis tools needed to establish the detector parameters and the detector R\&D needed to establish the future generation of neutrino experiments. A summary of the conclusions from the Detector Working Group from the International Scoping Study (ISS) for Future Neutrino Oscillation Facilities will be given. Special emphasis shall be made on recent developments in the description of a future 50 kton Magnetised Iron Neutrino Detector (MIND) and Totally Active Scintillator Detectors (TASD), which are the main candidate detectors to measure the wrong-sign muon golden channel at a Neutrino Factory. The Emulsion Cloud Chamber (ECC) is considered as the most promising detector to measure the $\tau$ appearance silver channel at a neutrino factory, which would allow to disentangle some of the degeneracies associated with the analysis of the neutrino oscillation signal. For a Super-beam or Beta-beam facility, a large scale $\sim 0.5$ Mton water Cherenkov detector is considered as the baseline detector, with other advanced detector concepts such as a Liquid Argon also under study. The article concludes with a roadmap for defining the design of future neutrino oscillation facilities by 2012, in order to be able to construct such a facility by the second half of the next decade.

10th International Workshop on Neutrino Factories, Super beams and Beta beams June 30 - July 52008

Valencia, Spain

\footnotetext{
${ }^{*}$ Speaker.

${ }^{\dagger}$ Support from the Science and Technology Facilities Council (U.K.) is gratefully acknowledged.
} 


\section{Introduction}

The weak neutrino eigenstates $\left(v_{e}, v_{\mu}, v_{\tau}\right)$ do not have to coincide with neutrino mass eigenstates $\left(v_{1}, v_{2}, v_{3}\right)$. Ignoring Majorana phases, the PMNS (Pontecorvo-Maki-Nakagawa-Sakata) neutrino mixing matrix $V_{P M N S}$, similar to the CKM matrix for quarks [1, 2], describes the mixture amongst neutrino states:

$$
V_{P M N S}=\left(\begin{array}{ccc}
1 & 0 & 0 \\
0 & c_{23} & s_{23} \\
0 & -s_{23} & c_{23}
\end{array}\right)\left(\begin{array}{ccc}
c_{13} & 0 & s_{13} e^{-i \delta} \\
0 & 1 & 0 \\
-s_{13} e^{i \delta} & 0 & c_{13}
\end{array}\right)\left(\begin{array}{ccc}
c_{12} & s_{12} & 0 \\
-s_{12} & c_{12} & 0 \\
0 & 0 & 1
\end{array}\right)
$$

with $c_{i j}=\cos \theta_{i j}$ and $s_{i j}=\sin \theta_{i j}$. The mixing angles $\theta_{12}$ and $\theta_{23}$, and the mass squared differences $\Delta m_{21}^{2}=m_{2}^{2}-m_{1}^{2}$ and $\left|\Delta m_{32}^{2}\right|=\left|m_{3}^{2}-m_{2}^{2}\right|$ are known (see Table 1) [4].

Table 1: Summary of the known PMNS parameters ( $2 \sigma$ errors).

\begin{tabular}{|l|c|c|c|c|c|}
\hline Parameters & $\Delta m_{31}^{2}\left(\mathrm{eV}^{2}\right)$ & $\Delta m_{21}^{2}\left(\mathrm{eV}^{2}\right)$ & $\sin ^{2} \theta_{23}$ & $\sin ^{2} \theta_{12}$ & $\sin ^{2} \theta_{13}$ \\
\hline Values & $\left(2.4_{-0.3}^{+0.3}\right) \times 10^{-3}$ & $\left(7.6_{-0.3}^{+0.5}\right) \times 10^{-5}$ & $0.50_{-0.12}^{+0.13}$ & $0.32_{-0.04}^{+0.05}$ & $\leq 0.033$ \\
\hline
\end{tabular}

The parameters $\theta_{13}$, the CP violating phase $\delta$ and the sign of $\Delta m_{32}^{2}$, which defines the neutrino mass hierarchy, are not known. The determination of these parameters are the main goals in neutrino oscillation physics over the coming years.

The neutrino oscillation probability at a distance $L$ of the golden channel $\left(v_{e} \rightarrow v_{\mu}\right.$ or $\left.\bar{v}_{e} \rightarrow \bar{v}_{\mu}\right)$ in matter (MSW effect) may be parameterised by [3]: $P_{v_{e} v_{\mu}\left(\bar{v}_{e} \bar{v}_{\mu}\right)}\left(\theta_{13}, \delta\right)=P_{1}+P_{2}+P_{3}+P_{4}$, with

$$
\begin{aligned}
& P_{1}=s_{23}^{2} \sin ^{2} 2 \theta_{13}\left(\frac{\Delta_{13}}{B_{\mp}}\right)^{2} \sin ^{2}\left(\frac{B_{\mp} L}{2}\right) \\
& P_{2}=c_{23}^{2} \sin ^{2} 2 \theta_{12}\left(\frac{\Delta_{12}}{A}\right)^{2} \sin ^{2}\left(\frac{A L}{2}\right) \\
& P_{3}=J \cos \delta \cos \left(\frac{\Delta_{13} L}{2}\right)\left(\frac{\Delta_{12}}{A} \frac{\Delta_{13}}{B_{\mp}}\right) \sin \left(\frac{A L}{2}\right) \sin \left(\frac{B_{\mp} L}{2}\right) \\
& P_{4}= \pm J \sin \delta \sin \left(\frac{\Delta_{13} L}{2}\right)\left(\frac{\Delta_{12}}{A} \frac{\Delta_{13}}{B_{\mp}}\right) \sin \left(\frac{A L}{2}\right) \sin \left(\frac{B_{\mp} L}{2}\right)
\end{aligned}
$$

where $\Delta_{i j}=\Delta m_{i j}^{2} / 2 E, B_{ \pm}=\left|A \pm \Delta_{13}\right|$, and $A$ is the matter parameter defined by $A=\sqrt{2} G_{F} n_{e}$, with $G_{F}$ and $n_{e}$ the Fermi coupling constant, and number density of electrons in the earth.

At the so-called "Magic Baseline", where $\frac{A L}{2}=\pi$, which corresponds to a baseline of $L \approx$ $7300-7600 \mathrm{~km}$, the terms $P_{2}, P_{3}$ and $P_{4}$ vanish and one obtains a clean determination of $\theta_{13}$. However, there are up to eight degeneracies and correlations between variables that need to be determined. The best strategy is to carry out a number of different experiments at different baselines and energies to solve the degeneracies. 


\section{International Scoping Study}

The International Scoping Study (ISS) looked at the physics, accelerator and detector prospects for future neutrino oscillation facilities to determine the remaining unknown oscillation parameters. The outcomes have been published as three reports $[5,6,7]$. The ISS compared the physics performance and detector requirements for three possible facilities:

- Super-beams: high powered (1-4 MW proton power) conventional neutrino beams from the decay of pions [8];

- Neutrino Factories: neutrino beams from the decay of muons [9]; and

- Beta beams: neutrino beams from the decay of radioactive isotopes [10].

Additionally, the ISS defined the scope of the accelerator parameters for a Neutrino Factory and Beta beam facilities (in collaboration with the Eurisol Design Study Beta beam group [11]).

Neutrino Factories produce neutrinos from muon decays in a storage ring. The rate is calculable by the kinematics of the decay (Michel spectrum). The International Scoping Study recommended a final muon energy of $25 \mathrm{GeV}$, which has been adopted by the Neutrino Factory International Design Study (IDS) [12], and can be shown in Figure 1. The design contains two muon storage rings, so that two different detectors at two different baselines can be accommodated to remove any degeneracies and correlations between variables.

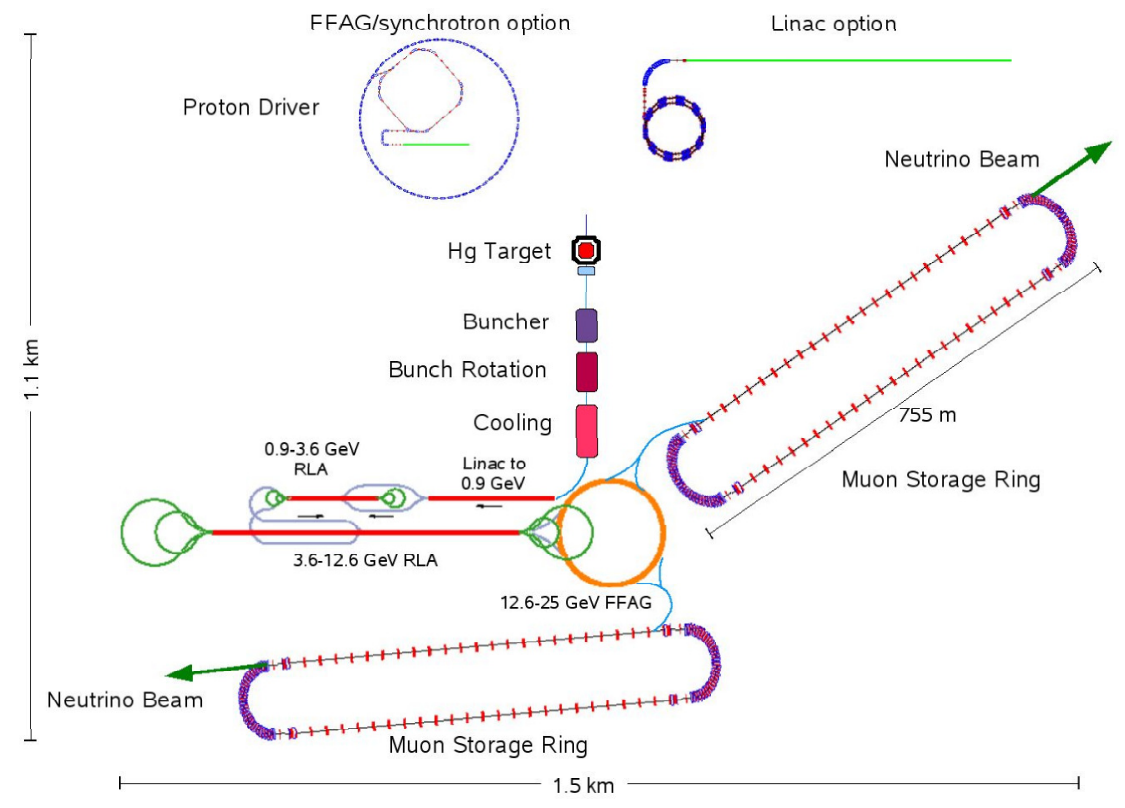

Figure 1: Schematic of the baseline specification for the Neutrino Factory International Design Study [12].

Since $v_{\mu}$ and $\bar{v}_{e}$ (or $\bar{v}_{\mu}$ and $v_{e}$ ) are produced simultaneously, this means that we need to determine the charge and lepton identity of the oscillation signal to separate from background. Hence, the detector requirements at a neutrino factory mandate a magnetic detector with good muon 
identification. Additionally, the neutrino factory has to provide sufficient neutrino luminosity $\left(10^{21}\right.$ muon decays year) to perform $\mathrm{CP}$ violation experiments at very long baselines. Two detectors at $4000 \mathrm{~km}$ and $7500 \mathrm{~km}$ were adopted by the ISS as the default option [7] to solve degeneracies and correlations between variables in the oscillation signals.

At a neutrino factory facility, the detector chosen was the Magnetised Iron Neutrino Detector (MIND), with a fiducial mass of $50 \mathrm{kton}$. This detector has the capability to carry out the $v_{\mu}$ "wrong-sign muon" appearance signal (the golden channel at a neutrino factory) [13] and will be discussed in more detail in the following section. The addition of a Magnetised Emulsion Cloud Chamber (MECC) of $10 \mathrm{kton}[14,15]$, as in the OPERA experiment [16], at the medium baseline detector site can be used to search for wrong-sign $v_{\tau}$ appearance (silver channel) [17]. One can assume a factor of two bigger than OPERA (4.1 kton of emulsion-iron target) consisting of 60 walls of emulsion, with 1.1 million emulsion-iron bricks. Candidate events are chosen by searching for a displaced vertex from the muon candidate. This experiment would reduce the correlation errors in the neutrino oscillation parameter space and can be used to search for new physics.

Beyond the default detector options, improvements at a Neutrino Factory include detectors with the capability for detecting the $v_{e}$ appearance (platinum) channels. These detectors also benefit from lower detection thresholds for the $v_{\mu}$ appearance channels, with the potential of observing the second oscillation maximum, which would provide improved accuracy in determining the neutrino oscillation parameters. Magnetised Liquid Argon solutions between 10-100 kton [18], or a magnetised Totally Active Scintillating Detector (TASD) of between 20-30 kton [19, 20], are possible detector technologies that are currently undergoing intense R\& D.

The TASD consists of 3333 modules with an $\mathrm{X}$ and $\mathrm{Y}$ plane each module, and with each plane containing 1000 triangular slabs of scintillator with a $3 \mathrm{~cm}$ base, for a total of 6.7 million channels. Muon momenta can be measured between $100 \mathrm{MeV} / \mathrm{c}$ to $15 \mathrm{GeV} / \mathrm{c}$. With a magnetic field of $0.5 \mathrm{~T}$, one obtains a reconstructed position resolution of $4.5 \mathrm{~mm}$. Muon reconstructed efficiency is close to $100 \%$ above $1 \mathrm{GeV}$, with a threshold around $0.5 \mathrm{GeV}$. The muon charge misidentification probability is below $10^{-4}$ above $0.5 \mathrm{GeV}$. A first attempt at electron identification is reported in [19]. As highlighted in the ISS detector report [7], magnetisation of large volumes remains a technological challenge, but a superconducting transmission line (STL), a superconducting cable containing its own cryostat, could be a potential solution to this problem. Further R\& D and engineering studies are required to make this technology feasible. With a TASD it is possible to run at a low energy neutrino factory with muons of $\sim 4 \mathrm{GeV}$ [21]. A far detector at a distance of $1480 \mathrm{~km}$ can measure the mass hierarchy and the CP violating phase $\delta$ (for $80 \%$ of the values of $\delta$ ) with a $95 \%$ CL sensitivity down to values of $\sin ^{2} 2 \theta_{13} \sim 10^{-3}$.

Near detectors at a neutrino factory should be able to measure flux and energy of $v_{\mu}$ and $\bar{v}_{e}$. A high event rate of $\sim 10^{9} \mathrm{CC}$ events per year is expected in a $50 \mathrm{~kg}$ detector. The flux can be determined by the detection of inverse muon decay or other leptonic processes. Since the crosssections for these processes are calculable to high accuracy $(\sim 0.1 \%)$ the flux can be deduced. The measured flux in a near detector can be used to predict the flux at a far detector and make the oscillation measurement [22, 23]. Other goals include the measurement of neutrino cross-sections with unprecedented accuracy and a measurement of the charm cross-section to control systematics of the far detector associated to the main background to the wrong-sign muon oscillation signal. A semiconductor vertex detector seems to be the only viable option in a high intensity environment 
to measure charm events. Studies of Monolithic Active Pixel Sensors (MAPS), for example the newly developed Vanilla sensor [24], for a pixel vertex detector at the near detector are underway.

At Beta beams, the neutrino beam is derived from the beta decay of accelerated radioactive nuclei. In the original Beta beam concept [10], ${ }^{6} \mathrm{He}$ accelerated to a $\gamma \sim 100$ is used for $v_{e}$ production and ${ }^{18} \mathrm{Ne}$ accelerated to a $\gamma \sim 60$ is used for $\bar{v}_{e}$ production. Future production schemes include the use of high $\mathrm{Q}$ beta beams such as ${ }^{8} \mathrm{~B}$ or ${ }^{8} \mathrm{Li}[25,26]$. Detectors do not need to be magnetised since only one neutrino species is produced. For both Beta beams and Super-beams, a very large ( $\sim 0.5$ Mton) Water Cherenkov detector is considered to be the baseline. A non-magnetised Liquid Argon or Totally Active Scintillating Detector like Nova are also considered.

\section{Magnetised Iron Neutrino Detector (MIND)}

The golden channel at a neutrino factory consists of observing "wrong-sign muons" in a magnetised iron calorimeter between 3000 and $7500 \mathrm{~km}$. In the original paper [13], the authors optimised the analysis for small values of $\theta_{13}$, so the efficiency at low energy was cut severely. The analysis, carried out using a fast simulation with parameterised detector settings, was recently revisited [27] and included in the ISS detector report. An improved event selection was chosen but perfect pattern recognition was still considered, with a reconstruction based on the detector parameterisation and including a dipole field instead of a toroidal field. The energy of fully contained muons $\left(E_{\mu}\right)$ was calculated by range, and that of scraping muons was determined by curvature. The hadron shower was parameterised as $\delta E_{\text {had }} / E_{\text {had }}=0.55 / \sqrt{E_{\text {had }}}$ and the neutrino energy was $E_{v}=E_{\mu}+E_{\text {had }}$. A kinematic analysis to eliminate background was carried out by exploiting the absolute muon momentum $P_{\mu}=\left|P_{\mu}\right|$ and the variable $Q_{t}=P_{\mu} \sin ^{2} \theta$, where $\theta$ is the angle between the lepton and the remaining hadron jet vector. Cuts in the $E_{v}-P_{\mu}$ and $E_{v}-Q_{t}$ planes remove the main backgrounds from hadron decay (charm decay in charged current and pion decay in neutral current interactions). The improved MIND event selection relied on a cut on the length of the muon track with respect to the final hadron $\left(L_{\mu}-L_{\text {had }}>150 \mathrm{~cm}\right)$ that reduces the charge misidentification background to $10^{-3}$. The signal efficiency reaches a plateau of $\sim 70 \%$ above $5 \mathrm{GeV}$ (Figure 2). Further improvements to this analysis, including full pattern recognition, are reported in [28].
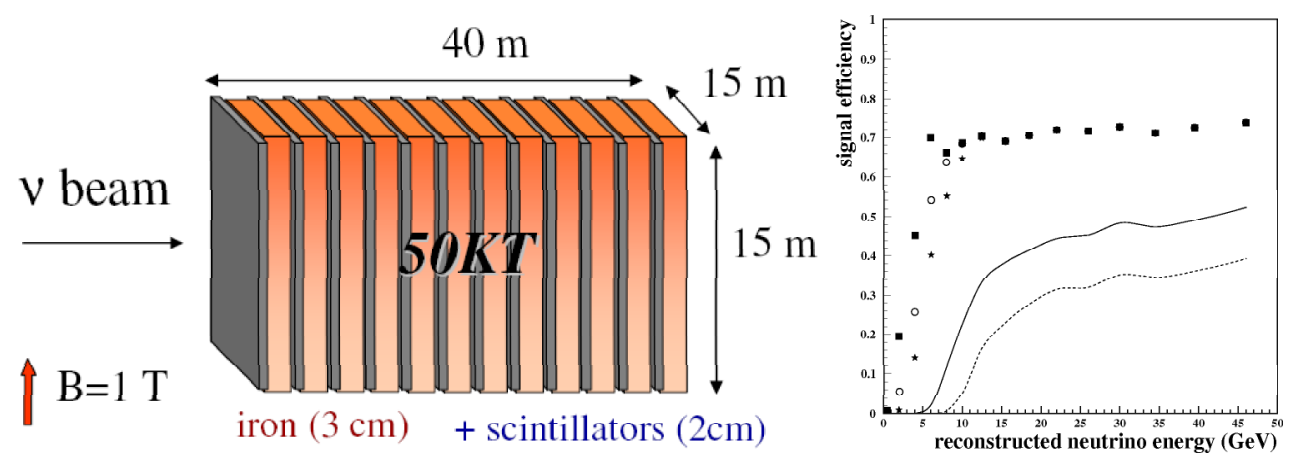

Figure 2: Schematic of MIND (left) and muon efficiency from golden channel analysis (points) compared to the efficiency from the old MIND analysis (lines) for different analysis cuts (left). 
The performance of the Neutrino Factory IDS baseline, which includes two MIND-like detectors, one at $4000 \mathrm{~km}$ and one at $7500 \mathrm{~km}$, is shown in Figure 3, for $3 \sigma$ contours [12]. Some of the R\& D needed for MIND includes the development of extruded scintillator bars (pioneered at Fermilab), readout fibres, high quantum efficiency photon detectors (Avalanche Photo Diodes, or Multi-pixel Photon Counters) and readout electronics. Further engineering work is needed to be able to design the magnetic field configuration, and for prototyping of small scale modules $\left(\sim 10^{-3}\right.$ of a full MIND, of dimensions $\sim 2 \times 2 \times 4 \mathrm{~m}^{3}$ ) for inclusion in a test beam.
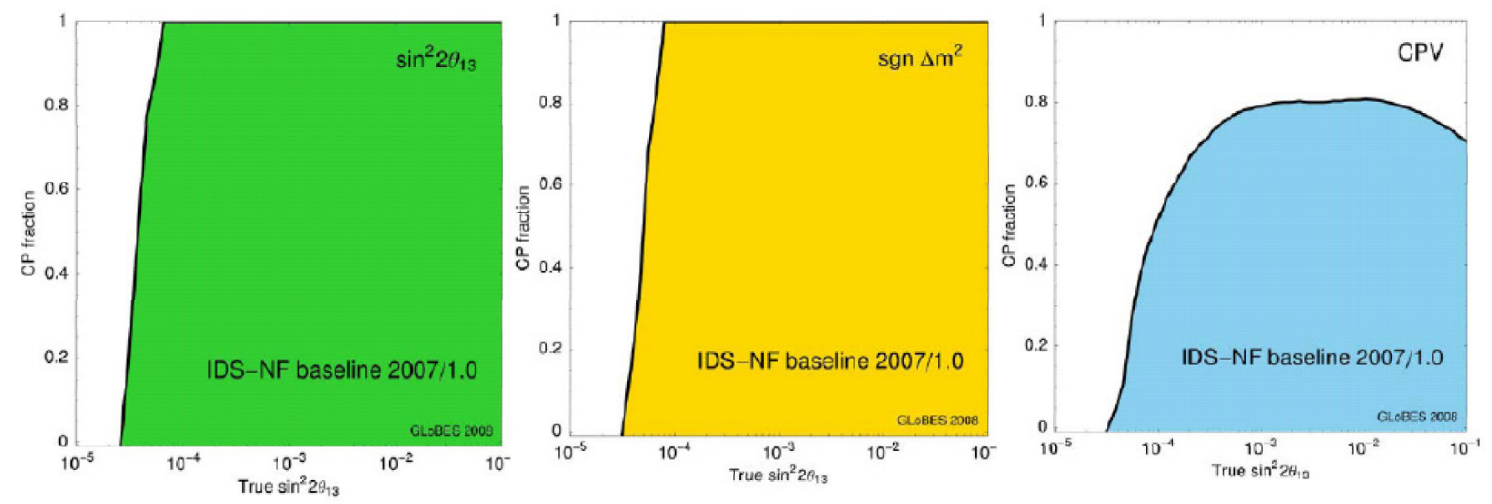

Figure 3: Performance sensitivity of the Neutrino Factory IDS baseline (two MIND detectors at 4000 and $7500 \mathrm{~km}$ ) for measuring $\theta_{13}$, the sign of $\Delta m_{32}^{2}$ and the CP violating phase $\delta$ at the $3 \sigma$ level.

The Indian Neutrino Observatory [29] is in an advanced stage of planning, has been recommended for funding and will be a $48 \times 16 \times 16 \mathrm{~m}^{3}$ magnetised iron calorimeter, read out by RPCs and with a $1.5 \mathrm{~T}$ magnetic field. While the main physics goal of this facility is the study of atmospheric neutrino oscillations with a magnetised detector, to search for matter effects, it could also constitute a far detector at a magic baseline for a neutrino beam either from Europe to India (CERN - INO $7152 \mathrm{~km}$ and RAL - INO $7653 \mathrm{~km}$ ) or from Japan to India (JPARC - INO $6556 \mathrm{~km}$ ).

Further improvements to the MIND analysis include a full GEANT4 simulation, with digitisation of hits, reconstruction, pattern recognition and the addition of a neutrino event generator with Quasi-Elastic (QE), Resonance (RES) and Deep Inelastic (DIS) neutrino events. The main goals are to demonstrate that for $E_{v}<10 \mathrm{GeV}$, the backgrounds can be kept below $10^{-3}$ and the efficiency can be increased with respect to previous analyses. This will allow to optimise the segmentation and the magnetic field, taking into account feasibility and cost. This work has already started, by implementing a Kalman filter with full pattern recognition [28], rejecting background due to charge misidentification, and rejecting hard scatters by implementing a kink finding algorithm (the remaining charge misidentification background is due to non-Gaussian multiple scattering).

\section{4. $R \&$ D plans in Europe}

EuroNu is a four year EU Design Study for "A High Intensity Neutrino Oscillation Facility in Europe". It will study super-beams, neutrino factory, beta-beams, neutrino detectors for the previous facilities and will perform a physics performance comparison amongst the facilities. As part of the neutrino detector work package, EuroNu aims to study the performance of the Magnetised 
Neutrino Iron Detector (MIND) for the golden measurement at a neutrino factory, a $\sim 0.5$ Mton water Cherenkov detector for beta and super beams and near detectors for all facilities. Further R\&D efforts are needed to develop and prototype different detector technologies. The Neutrino Factory IDS is a viable forum to coordinate the activity between Europe and other regions. The main goal is to develop test beams for neutrino detector R\& D at CERN and other laboratories and to construct prototypes to put into these beams to test technical feasibility and to optimise the detector properties.

Finally, EuCARD is an FP7 Integrating Activity proposal for "European Coordination for Accelerator Research and Development". It includes European R\& D on accelerator technologies, including a networking activity to define the next neutrino accelerator facilities by 2012 (called NEU2012). This aims to be the successor to the very successful BENE network that brought together European researchers studying future neutrino facilities.

\section{Conclusions and Neutrino Factory Roadmap}

The ISS studied all options for future neutrino facilities and narrowed the list of detector options for each facility. The launch of the Neutrino Factory International Design Study (IDS) has followed from this initiative, with the goal of developing an internationally agreed baseline for the Neutrino Factory accelerator complex and for the Neutrino Factory neutrino detector systems. The aim is to produce a Reference Design Report (RDR) for the Neutrino Factory by 2012. The main emphasis of the RDR will be on engineering, to evaluate the technical feasibility and cost of a future neutrino factory, necessary before planning and construction of the project can commence.

The next generation of reactor and accelerator based neutrino oscillation experiments (Double Chooz, T2K, Daya Bay and Nova) will reach sensitivity to $\sin ^{2} 2 \theta_{13} \sim 10^{-2}$ by 2012 . Knowledge on the value of $\theta_{13}$ and technical feasibility will dictate the high intensity neutrino facility required for high-precision neutrino oscillation measurements and discovery of CP violation in the neutrino sector. The main goal is to realise this facility by the second half of the next decade.

\section{References}

[1] Pontecorvo, B., Zh. Eksp. Teor. Fiz. 53, 1717 (1967) [Sov. Phys. JETP 26, 984 (1968)].

[2] Maki, Z., Nakagawa, M., and Sakata, S., Prog. Theor. Phys. 28, 870 (1962).

[3] Minakata, H. and Nunokawa, H. (2001), JHEP 0110001.

[4] T. Schwetz, AIP Conf.Proc. 981, 8-12, (2008). arXiv:0710.5027 [hep-ph].

[5] A. Bandyopadhyay et al., ISS Physics Working Group, Physics at a future Neutrino Factory and super-beam facility, RAL-TR-2007-19, arXiv:0710.4947 [hep-ph] (2007).

[6] J.S. Berg et al., ISS Accelerator Working Group, Accelerator design concept for future neutrino facilities, RAL-TR-2007-23, arXiv:0802.4023 [physics.acc-ph] (2008).

[7] T. Abe et al., ISS Detector Working Group, Detectors and flux instrumentation for future neutrino facilities, RAL-TR-2007-24, arXiv:0712.4129 [physics.ins-det] (2007).

[8] M. Zito, Baseline scenario for Super Beam Proton Driver, challenges and synergies with other programmes, in proceedings of 10th International Workshop on Neutrino Factories, Super beams and Beta beams (NuFact08), June 30 - July 5 2008, Valencia, Spain PoS (NUFACT08) [ 071 ] . 
[9] S. Geer, Phys. Rev. D57, 6989-6997 (1998). Erratum-ibid. D59, 039903 (1999).

[10] P. Zucchelli, Phys. Lett. B, 532 (2002), 166-172.

[11] Eurisol Design Study, http://www.eurisol.org/

[12] Neutrino Factory International Design Study, http://www.ids-nf.org/

[13] A. Cervera et al., Nucl. Phys. B 579, (2000), 17-55. Erratum-ibid. B593, (2001), 731-732.

[14] D.Autiero et al., Eur. Phys. J. C33, (2004), 243.

[15] L. Scotto Lavina, Design and performance of ECC-MECC, in proceedings of IOth International Workshop on Neutrino Factories, Super beams and Beta beams (NuFact08), June 30 - July 5 2008, Valencia, Spain POS (NUFACT08) [049].

[16] A. Cazes Experience from OPERA, in proceedings of 10th International Workshop on Neutrino Factories, Super beams and Beta beams (NuFact08), June 30 - July 5 2008, Valencia, Spain POS (NUFACT08) [048].

[17] A. Donini, D. Meloni and P. Migliozzi, Nucl. Phys. B 646, (2002),321-349.

[18] A. Ereditato, A. Rubbia, 7th International Workshop on Neutrino Factories and Superbeams (NuFact 05), Frascati, Italy, 21-26 Jun 2005. Nucl. Phys. Proc. Suppl. 155, (2006), 233-236.

[19] A. Bross, Status of TASD, in proceedings of 10th International Workshop on Neutrino Factories, Super beams and Beta beams (NuFact08), June 30 - July 5 2008, Valencia, Spain POS (NUFACT08) [043].

[20] M. Ishitsuka, Prospects for the platinum mode, in proceedings of 10th International Workshop on Neutrino Factories, Super beams and Beta beams (NuFact08), June 30 - July 5 2008, Valencia, Spain POS (NUFACT08) [050].

[21] A.D. Bross et al., Phys. Rev. D77, 093012, (2008). arXiv:0709.3889 [hep-ph].

[22] A. Laing, F.J.P. Soler, AIP Conf. Proc. 981, 166-168, (2008).

[23] A. Laing, F.J.P. Soler, Oscillation probability fits using Near Detector data at a Neutrino Factory, in proceedings of 10th International Workshop on Neutrino Factories, Super beams and Beta beams (NuFact08), June 30 - July 5 2008, Valencia, Spain PoS (NUFACT08) [129] .

[24] A. Blue et al., 11th Vienna Conference on Instrumentation (VCI 2007), Vienna, Austria, 19-24 Feb 2007. Nucl. Instrum. Meth. A581, 287-290, (2007).

[25] E. Wildner, Beta beam R\&D Prospects, in proceedings of 10th International Workshop on Neutrino Factories, Super beams and Beta beams (NuFact08), June 30 - July 5 2008, Valencia, Spain POS (NUFACT08) [007].

[26] C. Rubbia et al., Nucl. Instr. and Meth. A 568 (2006), 475-487.

[27] A. Cervera-Villanueva, AIP Conf. Proc. 981, (2008), 178-180.

[28] A. Cervera, A. Laing, Status of MIND, in proceedings of 10th International Workshop on Neutrino Factories, Super beams and Beta beams (NuFact08), June 30 - July 5 2008, Valencia, Spain POS (NUFACT08) [042].

[29] A. Raychaudhuri, Status of INO, in proceedings of 10th International Workshop on Neutrino Factories, Super beams and Beta beams (NuFact08), June 30 - July 5 2008, Valencia, Spain POS (NUFACT08) [045]. 